\title{
A novel missense mutation in the SLC26A4 gene in a chinese family with enlarged vestibular aqueducts
}

\section{Xiaohui He}

Xi 'an People's Hospital (Xi 'an Fourth Hospital)

\section{Shaozhi Zhao}

Xi 'an People's Hospital (Xi 'an Fourth Hospital)

\section{Lin Shi}

Xi 'an People's Hospital (Xi 'an Fourth Hospital)

\section{Yitong Lu}

Xi 'an People's Hospital (Xi 'an Fourth Hospital)

\section{Yintong Yang}

Xi 'an People's Hospital (Xi 'an Fourth Hospital)

Xinwen Zhang ( $\nabla$ xianxinwen@163.com )

Xi 'an People's Hospital (Xi 'an Fourth Hospital)

\section{Research Article}

Keywords: enlarged vestibular aqueducts, SLC26A4, A novel missense mutation, c.2069T>A

Posted Date: February 8th, 2022

DOI: https://doi.org/10.21203/rs.3.rs-1268645/v1

License: (c) (1) This work is licensed under a Creative Commons Attribution 4.0 International License. Read Full License 


\section{Abstract}

Background: SLC26A4 gene is closely related to enlarged vestibular aqueducts. Its homozygous mutation or compound heterozygous mutation may cause deafness and seriously affect the patient's life. As far as we know, this is the first reported pathogenic or probable pathogenic for the missense mutation. Our studies expand the mutation site of SLC26A4 gene and provide genetic counseling for the patient's family.

Methods: The patients who came to our hospital for hearing test and accompanied by bilateral hearing abnormalities were collected for 15 genetic tests for hereditary deafness. Those who are positive on chip screening will be verified by first-generation sequencing, combined with family history, hearing test, and computerized tomography (CT) of the temporal bone to diagnose the enlarged vestibular aqueducts. If necessary, perform second-generation sequencing.

Results: The patient failed the two hearing screenings on both sides, computerized tomography diagnosed enlarged vestibular aqueduct $(>1.5 \mathrm{~mm})$. Two pathogenic mutations, c.1174A $>\mathrm{T}$ and c.2069T>A were detected in SLC26A4. The heterozygous c.2069T>A mutation of SLC26A4 gene is a novel missense mutation that has been submitted to the clinvar website, Variation ID $₫ 1048780$.

Conclusion: Our findings enriched SLC26A4 gene spectrum and support previous studies that the enlarged vestibular aqueducts can be caused by a compound heterozygous mutation in SLC26A4.

\section{Background}

Hearing loss is one of the most serious sensory defects, which not only affects the quality of life, but also affects physical and mental health ${ }^{[1]}$. Many patients with hearing abnormalities have the phenotype of inner ear malformation. Among them, the enlarged vestibular aqueduct (EVA) is the most common one. In children, inner ear malformations associated with sensorineural hearing loss are the most common ${ }^{[2]}$ EVA patients have non-syndromic hearing loss, and a small number of patients with goiter are called Pendred syndrome (PDS) ${ }^{[3]}$. The PDS gene can cause Pendred syndrome (PS) and non-syndromic deafness. It was later renamed SLC26A4 and was found and located on chromosome $7 q^{[4]}$. This gene contains 21 exons and encodes the protein pendrin, which is expressed in the thyroid, kidney and inner ear, and plays a role in the transport of anions between the thyroid and inner ear ${ }^{[5]}$. The phenotype is the developmental malformation of the temporal bone, including large vestibular aqueduct syndrome (LVAS) and Mondini malformation.

Here, we report a case of sensorineural hearing loss in our hospital. Through bilateral hearing detection and CT imaging of the temporal bone, we made a preliminary diagnosis of the patient. In order to further investigate genetic factors, fifteen genetic tests for hereditary deafness were implemented. The proband and her mother c.1174A>T heterozygous mutation in the SLC26A4 gene, the father is wild-type at fifteen 
locus. There was no abnormality in the hearing of the parents. In order to further identify the cause, the patient underwent whole-exome sequencing and Sanger sequencing to verify his parents.

\section{Proband And Method}

\subsection{Clinical symptoms of the proband}

The proband is a three-year-old girl. Because there is no obvious cause for hearing loss in both ears, symptoms recurring, and sometimes tinnitus" was admitted to the hospital. On physical examination, the double external auditory canals were unobstructed, there was no viscous secretion, and the tympanic membrane was normal. Denies the history of surgical trauma, and denies the history of hypertension, diabetes, and heart disease. The first hearing screening failed, the second hearing screening (December $11,2018)$ failed in the left ear and passed in the right ear. The patient failed bilateral hearing screening on April 2, 2019 and had diagnosed by CT on May 14, 2019.The image findings: (1) Inner ear: bilateral internal auditory canals are symmetrical without enlargement and stenosis, bilateral vestibules are full, vestibular aqueduct is enlarged, the right side is about $4.1 \mathrm{~mm}$ wide, the left side It is about $4.4 \mathrm{~mm}$ wide, the spiral tubes of the bilateral cochlea appear to be a circle and a half, the semicircular canal structure is clear, and the shape is not abnormal. There is no high position of the bilateral jugular bulbs. (2) Middle ear: The bilateral mastoid processes are well developed, with gasification shape, no abnormal density, no abnormal density shadow in the mastoid sinus and tympanic sinus, no enlargement of the tympanum, and clear structure of the ossicles without damage. (3) The outer ear canal is unobstructed, and small flake-like high-density shadows can be seen in it. The diagnostic opinion is that bilateral vestibular aqueducts are dilated, except for cochlear spiral duct malformations. There is no family history, and the parents have no abnormal hearing.

\section{Genetic testing methods}

In order to further confirm the diagnosis, after the patient and her parents have signed the informed consent form, 15 genetic tests for hereditary deafness will be performed. To further find out the cause, the patient underwent whole-exome sequencing and Sanger sequencing to verify his parents.

\section{Result}

\subsection{Results of genetic testing}

The patient and their parents underwent fifteen genetic testing for hereditary deafness, and the results are shown in Figure.1.

The patient and her mother c.1174A>T heterozygous mutation in the SLC26A4 gene, the father is wildtype at fifteen locus. Because the patient's mother was pregnant again, in order to find out the cause of the patient's hearing abnormalities, the Proband performed whole-exome sequencing and verified his parents using Sanger sequencing. At the same time, the patient's father performed the first-generation 
sequencing of the whole SLC26A4 gene. The results of the proband are shown in Table.1, and the firstgeneration sequencing verification results are shown in Figures. 2 and Figures.3.

\subsection{The pathogenicity rating of the variant site}

According to American College of Medical Genetics and Genomics (ACMG) guidelines, SLC26A4:NM_000441.2:exon10:c.1174A>T:p.N392Y is classified as pathogenic by fulfilling the standard PM3_VeryStrong, PM1, PP3, PP4, PS3_Supporting, PM2_Supporting.

Table.1 The results of whole-exome sequencing and Sanger sequencing

\begin{tabular}{|c|c|c|c|c|}
\hline gene & Mutation location & $\begin{array}{l}\text { Gene } \\
\text { subregion }\end{array}$ & HGVS & Heterozygosity \\
\hline \multirow[t]{3}{*}{ SLC26A4 } & $\begin{array}{l}\text { chr7:107690148- } \\
107690148\end{array}$ & exon10 & $\begin{array}{l}\text { NM_000441.2 :c.1174 } \\
\text { A>T:p.N392Y }\end{array}$ & $\begin{array}{l}\text { Proband } \mathbb{} \\
\text { Heterozygous }\end{array}$ \\
\hline & & & & Father囚wild \\
\hline & & & & $\begin{array}{l}\text { Mother } \ \\
\text { Heterozygous }\end{array}$ \\
\hline \multirow[t]{3}{*}{ SLC26A4 } & $\begin{array}{l}\text { chr7:107704365- } \\
107704365\end{array}$ & exon18 & $\begin{array}{l}\text { NM_000441.2:c.2069 } \\
\text { T>A:p.V690E }\end{array}$ & $\begin{array}{l}\text { Proband } \rrbracket \\
\text { Heterozygous }\end{array}$ \\
\hline & & & & $\begin{array}{l}\text { Father囚 } \\
\text { Heterozygous }\end{array}$ \\
\hline & & & & Mother》wild \\
\hline
\end{tabular}

According to the literature, Pathogenic or probable pathogenic variants detected in translocations of variants in 3 individuals with deafness ${ }^{[6]}$ (PM3_VeryStrong). The variant occurred in the functional structure of SLC26A/SulP transporter domain (PM1). Predicted by multiple statistical methods (REVEL), the results show that the mutation has harmful effects on genes or gene products (PP3). The corresponding disease of the variant is consistent with the phenotype of this case (PP4). It is reported in the literature that immunofluorescence experiments have confirmed that this mutation causes gene function damage ${ }^{[7]}$ (PS3_Supporting). This mutation is in the China Genome Database, the Human Exome Database (ExAC), the reference population Thousand Genome (1000G) and the Population Genome Mutation Frequency Database (gnomAD) are 0.00071531, 8.24198466990851e-06, 0.000199681 and 0.00019253 , respectively. This known variant is assessed as pathogenic in the ClinVar database. This known variant is assessed as DM in the HGMD database ${ }^{[8]-[11]}$ (PM2_Supporting).

According to the ACMG guidelines, SLC26A4:NM_000441.2:exon18:c.2069T>A:p.V690E is classified as likely pathogenic by fulfilling the criteria PM1, PM2, PM3, PP3, PP4.

The mutation occurred in the functional domain of the STAS domain (PM1). The mutation is in the China Genome Database, the Human Exome Database (ExAC), the Reference Population Thousand Genome (1000G) and the Population Genome Mutation Frequency Database (gnomAD) are not found (PM2). This 
variant forms a compound heterozygosity with the c.1174A>T mutation site in the SLC26A4 gene (PM3). Predicted by a variety of statistical methods (REVEL), the results show that the variant is Genes or gene products cause harmful effect (PP3). The corresponding disease of the variant matches the EVA phenotype of this case (PP4).

\section{Discussion And Conclusion}

It was found that the genetic results of this patient showed the c.1174A>T (p.N392Y) and c.2069T>A (p.V690E) mutations of SLC26A4. By consulting the literature and database, the c.2069T>A (p.V690E) mutation of SLC26A4 has not been reported, which forms a compound heterozygosity with the c. $1174 \mathrm{~A}>\mathrm{T}$ mutation site. The mutation occurred in the functional domain of the STAS domain (amino acid position 535-729). The variant may affect the protein function of SLC26A4.

Elucidating the influence of SLC26A4 gene function will have a certain guiding role in the later cochlear implantation or other treatments for deaf patients. And because SLC26A4 gene mutation screening can detect and diagnose LVAS during neonatal hearing screening, it has become an important supplementary method for temporal bone CT screening. Our research provides a basis for the patient's clinical diagnosis and genetic counseling. The patient's mother is pregnant again and her fetus is heterozygous at c.1174A>T:p.N392Y and wild type at c.2069T>A:p.V690E囚Figure 4囚. Genetic counseling recommends continuing pregnancy. After follow-up, the baby girl, four months old, had a normal routine physical examination and passed the newborn hearing screening. It also clarified the goal for the follow-up prenatal diagnosis. Enriching the mutation spectrum of the SLC26A4 gene has a complementary effect on the rare mutation sites of deafness. A total of 8647 mutations have been reported in SLC26A4, of which 487 are Pathogenic mutations, and Likely pathogenic mutations 118.

(https://deafnessvariationdatabase.org/gene/SLC26A4). Missense mutations accounted for the majority, followed by frameshift mutations and splice site mutations. High-throughput sequencing technology has significant advantages in identifying the genetic factors of diseases. At present, high-throughput sequencing of fetal free DNA in maternal peripheral blood has opened up a new development direction for non-invasive prenatal diagnosis of hereditary deafness, which will further promote genetics.

Pendrin is a membrane transport protein capable of exchanging anions between the cytoplasm and extracellular fluid. It can mediate chloride $\left(\mathrm{Cl}^{-}\right)$, hydroxide $\left(\mathrm{OH}^{-}\right)$, bicarbonate $\left(\mathrm{HCO}^{-}\right)$and iodide $\left(\mathrm{I}^{-}\right)$ exchange ${ }^{[12]}$. Studies have shown the role of two genes KCNJ10 and FOXI1 in PDS, and it has been suggested that double gene mutations, namely SLC26A4 and FOXI1 or KCNJ10 may cause Pendred syndrome ${ }^{[13]}$.

FOXI1 can bind SLC26A4 and mediate its activation and transcription ${ }^{[14]}$. However, many studies have shown that the relationship between KCNJ10 and FOXI1 and PDS is very small. It is very unlikely that these genes will be screened in patients with EVA. Other factors such as genetics or environment may play a more important role in the etiology of PDS/EVA ${ }^{[15]-[17]}$. 
Studies have shown that the transport function of SLC26A4 protein can be observed through cell experiments, so that the activity of SLC25A4 protein can be predicted ${ }^{[18]}$. In previous cell line studies, some SLC26A4-related mutants have been shown to cause disease through the transport process of pendrin rather than its expression level using HEK293 cells transfected ${ }^{[19]}$. The human gene SLC26A4 is homologous to the mouse Slc26a4, which encodes the protein pendrin. The study of this mouse model revealed the pathophysiological mechanism related to the loss of function or hypofunction mutation of SLC26A4 ${ }^{[20][21]}$. Reports in the literature show that transgenic mice for Slc26a4 variants have been constructed to mimic its pathogenic structure ${ }^{[22]}$. Reports show only $86 \%$ identity between mice and humans, and the amino acid sequence at the $\mathrm{C}$-terminus of pendrin is less conserved. The amino acid sequence conservation identity of the transmembrane domain of the protein is $92 \%$. Therefore, we speculate that the mouse lacking phenotype of the Slc26a4 C-terminal variant could be ascribed to the different protein structures of the $\mathrm{C}$-terminus of human and mouse pendrin. (https://www.uniprot.org/). The p.V690E variant is also C-terminal and does not seem feasible to construct a mouse model. We plan to focus on the function of p.V690E, transfect it into HEK293 cells to analyze its cellular localization and anion exchange activity, and verify the pathogenicity of this site through a series of functional tests.

\section{Abbreviations}

CT: computerized tomography; EVA: enlarged vestibular aqueduct; LVAS: large vestibular aqueduct syndrome; ACMG: American College of Medical Genetics and Genomics guidelines; PDS: Pendred syndrome.

\section{Declarations}

\section{Acknowledgements}

The authors would like to thank Xi'an Science and Technology Bureau for funding the study

\section{Authors' contributions}

XWZ supervised the work, YTY obtained the patient consent; XHH, SZZ and LS conducted genetic analysis of mutation sites; $\mathrm{XHH}$ conducted experiments and wrote the paper; YTL conducted protein structure analysis, and all authors participated and approved the manuscript the final version.

\section{Funding}

Science and Technology Program of Xi 'an (J201902043)

\section{Availability of data and materials}

The details of the variant analyzed during the current study are available in the ClinVar repository, under the Accession Number SCV001548357.1. (https://www.ncbi.nlm.nih.gov/clinvar/variation/1048780/? 
new_evidence=false) The raw datasets generated during the current study are not publicly available because it is possible that individual privacy could be compromised. It is possible to apply for permission to obtain access to the raw sequencing data and the details of the postmortem examination through the corresponding author.

\section{Ethics approval and consent to participate}

All methods were carried out in accordance with relevant guidelines and regulations. The studies involving human participants were reviewed and approved by Ethics Committee of Xi 'an People's Hospital (Xi 'an Fourth Hospital) (Xi 'an, China). Written informed consent to participate in this study was provided by the participants' legal guardian/next of kin.

\section{Consent for publication}

Written informed consent was obtained from the individual(s), and minor(s)' legal guardian/next of kin, for the publication of any potentially identifiable images or data included in this article..

\section{Competing interests}

The authors declare that they have no competing interests.

\section{Author details}

${ }^{1}$ Xi 'an People's Hospital (Xi 'an Fourth Hospital), Xi 'an, China

\section{References}

1. Brown C S, Emmett S D, Robler S K, et al. Global Hearing Loss Prevention[J]. Otolaryngologic Clinics of North America, 2018:S0030666518300069.

2. Griffith A J, Wangemann P. Hearing Loss Associated with Enlargement of the Vestibular Aqueduct: Mechanistic Insights from Clinical Phenotypes, Genotypes, and Mouse Models[J]. Hearing Research, 2011, $281(1-2): 11-17$.

3. JL Wémeau, Kopp P. Pendred syndrome[J]. Best Practice \& Research Clinical Endocrinology \& Metabolism, 2017:213.

4. Everett $L A$, Glaser $B$, Beck $J$ C, et al. Pendred syndrome is caused by mutations in a putative sulphate transporter gene (PDS)[J]. Nature Genetics, 1997, 17(4):411-422.

5. Aigerim, Bizhanova, Peter, et al. Genetics and phenomics of Pendred syndrome[J]. Molecular and Cellular Endocrinology, 2010, 322(1-2):83-90.

6. Huang S, Han D, Yuan Y, et al. Extremely discrepant mutation spectrum of SLC26A4 between Chinese patients with isolated Mondini deformity and enlarged vestibular aqueduct[J]. Journal of Translational Medicine, 9, 1(2011-09-30), 2011, 9(1):167-167. 
7. Ishihara K, Okuyama S, Kumano S, et al. Salicylate restores transport function and anion exchanger activity of missense pendrin mutations[J]. Hearing Research, 2010, 270(1-2):110-118.

8. Ideura M, Nishio S Y, H Moteki, et al. Comprehensive analysis of syndromic hearing loss patients in Japan[J]. Scientific Reports, 2019, 9(1).

9. Bassot C, Minervini G, Leonardi E, et al. Mapping pathogenic mutations suggests an innovative structural model for the pendrin (SLC26A4) transmembrane domain[J]. Biochimie, 2016:S0300908416302322.

10. Yu Y, Yang Y, Lu J, et al. Two Compound Heterozygous Were Identified in SLC26A4 Gene in Two Chinese Families With Enlarged Vestibular Aqueduct[J]. Clinical and experimental otorhinolaryngology, 2018, 12(1):50.

11. Qian L, Ji Y, Han B, et al. Comparative study of mutation spectrums of MT-RNR1 m.1555A>G, GJB2, and SLC26A4 between familial and sporadic patients with nonsyndromic sensorineural hearing loss in Chinese Han.[J]. Chinese Medical Journal, 2014, 127(018):3233-3237.

12. Soleimani $M$, Greeley T, Petrovic $S$, et al. Pendrin:an apical $\mathrm{Cl} / \mathrm{OH} / \mathrm{HCO}$ exchanger in the kidney cortex[J]. American Journal of Physiology Renal Physiology, 2001, 280(2):356-64.

13. Yang T, li J G G, Wu H, et al. Mutations of KCNJ10 Together with Mutations of SLC26A4 Cause Digenic Nonsyndromic Hearing Loss Associated with Enlarged Vestibular Aqueduct Syndrome[J]. American Journal of Human Genetics, 2009, 84(5):651-657.

14. Tao, Yang, and, et al. Transcriptional Control of SLC26A4 Is Involved in Pendred Syndrome and Nonsyndromic Enlargement of Vestibular Aqueduct (DFNB4)[J]. American Journal of Human Genetics, 2007.

15. Landa P, Differ A M, Rajput K, et al. Lack of significant association between mutations of KCNJ10 or FOXI1 and SLC26A4 mutations in pendred syndrome/enlarged vestibular aqueducts[J]. BMC Medical Genetics, 2013, 14(1):85-85.

16. Zhao X, Cheng X, Huang L, et al. Analysis of mutations in the FOXI1 and KCNJ10 genes in infants with a single-allele SLC26A4 mutation[J]. Bioscience trends, 2019, 13(3).

17. Chen K, Wang X, Sun L, et al. Screening of SLC26A4, FOXI1, KCNJ10, and GJB2 in bilateral deafness patients with inner ear malformation[J]. Otolaryngology-head and neck surgery: official journal of American Academy of Otolaryngology-Head and Neck Surgery, 2012, 146(6):972.

18. Moreno, Dossena, Botta, et al. Functional assessment of allelic variants in the SLC26A4 gene involved in Pendred syndrome and nonsyndromic EVA[J]. PNAS, 2008 Nov 25;105(47):18608-13.

19. Ishihara K, Okuyama $S$, Kumano $S$, et al. Salicylate restores transport function and anion exchanger activity of missense pendrin mutations[J]. Hearing Research, 2010, 270(1-2):110-118.

20. Wangemann P. Mouse Models for Pendrin-Associated Loss of Cochlear and Vestibular Function[J]. Cellular Physiology \& Biochemistry, 2013, 32(7):157-165.

21. Nakanishi H, Prakash P, Ito T, et al. Genetic Hearing Loss Associated With Autoinflammation[J]. Frontiers in Neurology, 2020, 11. 
22. Ying-Chang, Lu, Chen-Chi, et al. Differences in the Pathogenicity of the p.H723R Mutation of the Common Deafness-Associated SLC26A4 Gene in Humans and Mice[J]. PLoS ONE, 2013, 8(6):e64906.

\section{Figures}

\section{Figure 1}

The results of fifteen genetic tests for hereditary deafness

\section{Figure 2}

The results of Sanger sequencing A wild control sequence B, D The patient and her mother is c.1174A>T heterozygous mutation of SLC26A4 C The patient's father is wild-type

\section{Figure 3}

The results of Sanger sequencing E wild control sequence F, G The patient and her mother is c.2069T>A heterozygous mutation of SLC26A4 H The patient's mother is wild-type 


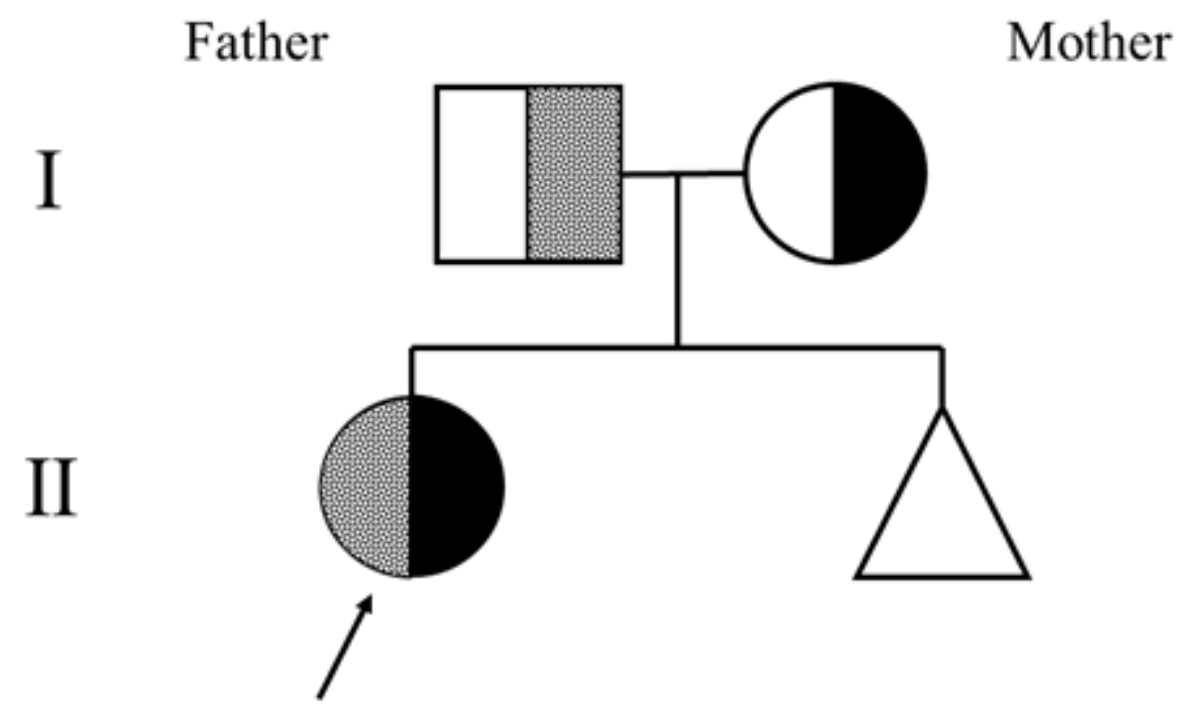

\section{Proband}

c. $1174 \mathrm{~A}>\mathrm{T}$ heterozygous mutation c.2069 T>A heterozygous mutation

Brother or Sister

c. $1174 \mathrm{~A}>\mathrm{T}$ heterozygous mutation

Figure 4

SLC26A4 mutations in the family with EVA 